\title{
Fluid overload and acute kidney injury: cause or consequence?
}

\author{
Marlies Ostermann ${ }^{1 *}$ D, Heleen M. Oudemans-van Straaten² and Lui G. Forni ${ }^{3,4}$ \\ See related research by Wang et al., http://www.ccforum.com/content/19/1/371
}

\begin{abstract}
There is increasing evidence that fluid overload and acute kidney injury (AKI) are associated but the exact cause-effect relationship remains unclear. Wang and colleagues analysed patients admitted to 30 intensive care units in China and found that fluid accumulation was independently associated with an increased risk of AKI and mortality. This commentary focuses on the close pathophysiological link between AKI and fluid overload and discusses the implications for clinical practice. It outlines some of the challenges, including the difficulty in diagnosing fluid overload reliably with current methods, and stresses the importance of personalised fluid therapy with physiological end-points to avoid the deleterious effects of fluid overload.
\end{abstract}

Fluids are the second most common intervention in acutely ill patients (after oxygen). Whilst the benefits of early fluid resuscitation in patients with shock and acute kidney injury (AKI) have been accepted, uncertainty remains about the optimal type, volume and timing of fluid delivery. There is growing evidence that fluid administration beyond the correction of hypovolaemia is associated with increased morbidity, a longer hospital stay and mortality. In a recent article in Critical Care, Wang et al. analysed the data of 2526 patients admitted to 30 intensive care units (ICUs) in China and showed that even relatively small degrees of fluid overload were independently associated with an increased risk of AKI and mortality [1]. Others have shown associations between fluid overload and poor outcomes in patients with acute lung injury, congestive heart failure, and AKI

\footnotetext{
* Correspondence: marlies.ostermann@gstt.nhs.uk

'Department of Critical Care and Nephrology, King's College London, Guy's \& St Thomas' Hospital, Westminster Bridge Road, London SE1 7EH, UK Full list of author information is available at the end of the article
}

receiving renal replacement therapy (RRT) and patients undergoing major surgery [2-6].

Although there is now little doubt that fluid overload is associated with AKI, it is difficult to disentangle the cause-effect relationship. Furthermore, translating the results into clinical practice is very challenging, especially since some important issues are still unresolved.

First, defining fluid overload is not straightforward. By convention, most studies in the literature, including the study by Wang et al. [1], defined fluid overload by a percentage increase in body weight from day of admission to the ICU. However, this assumes that patients were intravascularly replete on admission (which is often not the case). It also ignores any insensible losses as well as fluid administration in the pre-ICU setting.

Second, whether the consequences of fluid overload depend on the specific type of fluid has not been studied. It is plausible that fluid overload as a result of excessive crystalloid administration has a different impact compared with fluid accumulation following infusion of colloids or massive transfusion of blood products. More data are necessary. The timing of volume administration may also be relevant. A positive fluid balance of 5 litres over an initial 24-hour period followed by no further fluid gain may have a different outcome compared with the same net balance over a period of days.

Third, fluid overload may result from overzealous fluid administration or oliguria or a combination of the two. The differentiation is important since fluid overload caused by excessive fluid therapy is potentially avoidable whereas fluid overload as a result of oliguria may reflect AKI and may not be easily modifiable without RRT [7]. Most studies have not distinguished between the two. In a recent retrospective analysis, it was demonstrated that fluid administration, rather than low urine output, was independently associated with progression from AKI stage I to stage III [8]. Clearly, more detailed work is 
necessary to identify the exact factors responsible for fluid overload.

Finally, the fact that fluid overload is associated with AKI does not prove causality. One key problem is that the effects of volume overload and the effects of AKI are similar. Both lead to multi-organ dysfunction. They also often have the same denominator, including endothelial dysfunction due to inflammation or ischaemia/reperfusion injury with decay and shedding of the glycocalyx and subsequent capillary leakage [9]. Patients with more severe endothelial dysfunction tend to develop both fluid overload and AKI following fluid administration. Importantly, hypervolaemia may stretch the vascular wall and worsen vascular permeability, possibly by atrial natriuretic peptide-induced damage to the glycocalyx [10] (Fig. 1). Unpicking this vicious circle can be very difficult.

Nevertheless, the study by Wang et al. does confirm what other studies have shown previously that a positive fluid balance is associated with worse outcomes. The crucial question for clinical practice is how to ensure appropriate and timely correction of hypovolaemia without causing fluid overload. Unfortunately, the tools to guide fluid management and to inform the clinical team when fluid administration is no longer beneficial are inadequate. Central venous pressure (CVP) or dynamic techniques like fluid responsiveness or echocardiography are often used. However, the CVP does not accurately reflect intravascular volume status and can be misleading [11]. Measuring changes in pulse pressure and stroke volume may provide very useful information for assessing preload and fluid responsiveness but does not diagnose or quantify fluid overload either. Crucially, these techniques are not readily available outside critical care areas. Cumulative fluid balance gives an indication about the fluid input-output relationship but does not correct for non-measurable losses, is often inaccurate [12] and does not indicate whether a patient is intravascularly hypovolaemic, euvolaemic or overloaded.

In the absence of any sophisticated tools to confirm euvolaemia or diagnose fluid overload, initial fluid resuscitation should be guided by an assessment of fluid responsiveness followed by fluid therapy titrated against individualized end-points $[13,14]$. The passive leg raising (PLR) manoeuvre (i.e., internal fluid loading which is reversible) coupled with real-time stroke volume monitoring has been recommended to assess the need for fluid therapy [14].

There is some prospect that novel biomarkers may also have a role to prevent fluid overload. Mekontso Dessap et al. randomly assigned 304 mechanically ventilated patients to a strategy driven by B-type natriuretic peptide (BNP) or usual care [15]. Patients in the BNP-led cohort received diuretics and fluid restriction on days when their BNP was at least $200 \mathrm{pg} / \mathrm{ml}$. Fluid balance was more negative and time to successful extubation was shorter in this group.

\section{Conclusions}

Fluid overload, AKI and poor outcomes are clearly associated. However, whether AKI has caused fluid overload or vice versa can be difficult to determine. Better tools are necessary to measure intravascular volume status. Until then, fluid therapy needs to be personalised with physiological end-points, guided by dynamic tests like PLR manoeuvre, and monitored by using cumulative fluid balance coupled, where possible, with daily weights to avoid the adverse outcomes associated with fluid overload.

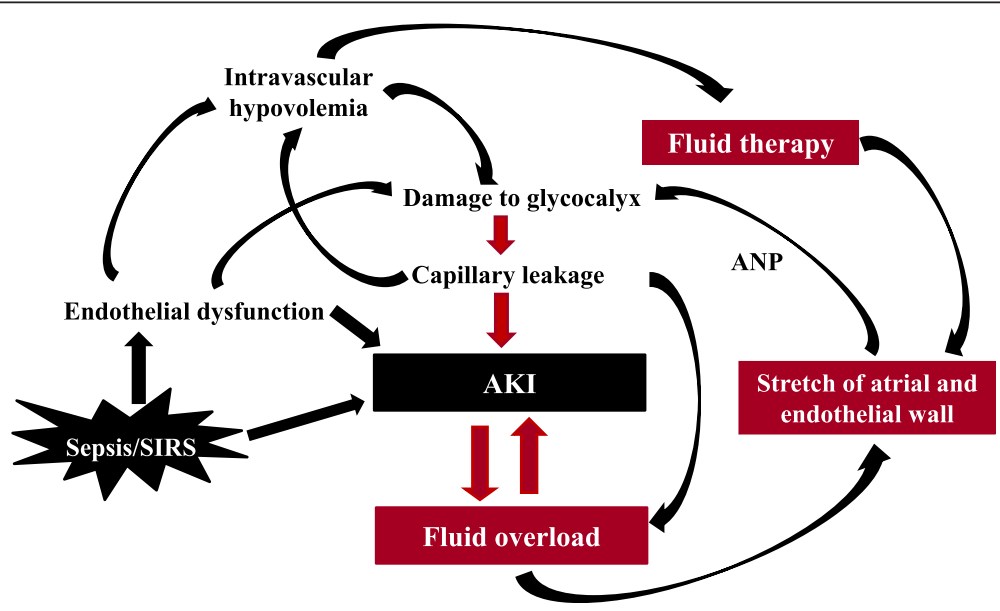

Fig. 1 Interconnected relationship between acute kidney injury and fluid overload. AKI acute kidney injury, ANP atrial natriuretic peptide, SIRS systemic inflammatory response syndrome 


\section{Abbreviations}

AKl: acute kidney injury; BNP: B-type natriuretic peptide; CVP: central venous pressure; ICU: intensive care unit; PLR: passive leg raising; RRT: renal replacement therapy.

\section{Competing interests}

The authors declare that they have no competing interests.

\section{Authors' contribution}

All authors read and approved the final manuscript.

\section{Author details}

'Department of Critical Care and Nephrology, King's College London, Guy's \& St Thomas' Hospital, Westminster Bridge Road, London SE1 7EH, UK. ${ }^{2}$ Department of Adult Intensive Care, VU University Medical Centre, De Boelelaan 1118, $1081 \mathrm{HZ}$ Amsterdam, The Netherlands. ${ }^{3}$ Surrey Perioperative Anaesthesia \& Critical Care Collaborative Research Group Royal Surrey County Hospital NHS Foundation Trust \& School of Health Sciences, Faculty of Health \& Medical Sciences, University of Surrey, Guildford GU2 7XH, UK. ${ }^{4}$ Intensive Care Unit, Royal Surrey County Hospital NHS Foundation Trust, Egerton Road, Guildford GU2 7XX, UK.

\section{Published online: 27 December 2015}

\section{References}

1. Wang N, Jiang L, Zhu B, Wen Y, Xi XM, The Beijing Acute Kidney Injury Trial (BAKIT) workgroup. Fluid balance and mortality in critically ill patients with acute kidney injury: a multicenter prospective epidemiological study. Crit Care. 2015;19:371.

2. Sakr Y, Vincent JL, Reinhart K, Groeneveld J, Michalopoulos A, Sprung CL, et al. High tidal volume and positive fluid balance are associated with worse outcome in acute lung injury. Chest. 2005;128:3098-108.

3. Vaara ST, Korhonen A-M, Kaukonen K-M, Nisula S, Inkinen O, Hoppu S, et al. Fluid overload is associated with an increased risk for 90-day mortality in critically ill patients with renal replacement therapy: data from the prospective FINNAKI study. Crit Care. 2012;16:R197.

4. RENAL Replacement Therapy Study Investigators, Bellomo R, Cass A, Cole L, Finfer S, Gallagher M, et al. An observational study fluid balance and patient outcomes in the randomized evaluation of normal vs. augmented level of replacement therapy trial. Crit Care Med. 2012;40:1753-60.

5. Bouchard J, Soroko SB, Chertow GM, Himmelfarb J, Ikizler TA, Paganini EP, et al. Fluid accumulation, survival and recovery of kidney function in critically ill patients with acute kidney injury. Kidney Int. 2009;76:422-7.

6. Payen D, de Pont AC, Sakr Y, Spies C, Reinhart K, Vincent JL, et al. A positive fluid balance is associated with a worse outcome in patients with acute renal failure. Crit Care. 2008;12:R74.

7. Rosner MH, Ostermann M, Murugan R, Prowle JR, Ronco C, Kellum JA, et al. Indications and management of mechanical fluid removal in critical illness. Br J Anaesth. 2014;113:764-71.

8. Raimundo M, Crichton S, Martin JR, Syed Y, Varrier M, Wyncoll D, et al. Increased fluid administration after early acute kidney injury is associated with less renal recovery. Shock. 2015;44:431-7.

9. Chappell D, Bruegger D, Potzel J, Jacob M, Brettner F, Vogeser M, et al. Hypervolaemia increases release of atrial natriuretic peptide and shedding of the endothelial glycocalyx. Crit Care. 2014;18:538.

10. Bruegger D, Jacob M, Rehm M, Loetsch M, Welsch U, Conzen P, et al. Atrial natriuretic peptide induces shedding of endothelial glycocalyx in coronary vascular bed of guinea pig hearts. Am J Physiol Heart Circ Physiol. 2005;289:H1993-9.

11. Marik PE, Cavallazzi R. Does the central venous pressure predict fluid responsiveness? An updated meta-analysis and a plea for some common sense. Crit Care Med. 2013;41:1774-81.

12. Perren A, Markmann M, Merlani G, Marone C, Merlani P. Fluid balance in critically ill patients. Should we really rely on it? Minerva Anestesiol. 2011;77:802-11.

13. Ogbu OC, Murphy DJ, Martin GS. How to avoid fluid overload. Curr Opin Crit Care. 2015;21:315-21.

14. Marik P, Bellomo R. A rational approach to fluid therapy in sepsis. Br J Anaesth. 2015 Oct 27. pii: aev349. [Epub ahead of print].

15. Mekontso Dessap A, Katsahian S, Roche-Campo F, Kouatchet A, Tomicic V, Beduneau $G$, et al. Natriuretic peptide-driven fluid management during ventilator weaning: a randomized controlled trial. Am J Respir Crit Care. 2012;186:1256-63. 